\title{
Breakfast habits among European adolescents and their association with sociodemographic factors: the HELENA (Healthy Lifestyle in Europe by Nutrition in Adolescence) study
}

Lena Hallström ${ }^{1,2, *}$, Carine A Vereecken ${ }^{3,4}$, Idoia Labayen ${ }^{2,5}$, Jonatan R Ruiz ${ }^{2}$, Cinzia Le Donne ${ }^{6}$, Magdalena Cuenca García ${ }^{7}$, Chantal C Gilbert $^{8}$, Sonia Gómez Martínez ${ }^{9}$, Eva Grammatikaki ${ }^{10}$, Inge Huybrechts ${ }^{4}$, Anthony Kafatos ${ }^{11}$, Mathilde Kersting ${ }^{12}$, Yannis Manios ${ }^{10}$, Dénes Molnár ${ }^{13}$, Emma Patterson ${ }^{14}$, Kurt Widhalm ${ }^{15}$, Tineke De Vriendt ${ }^{4}$, Luis A Moreno ${ }^{16}$ and Michael Sjöström², on behalf of the HELENA Study Group

${ }^{1}$ School of Health, Care and Social Welfare, Box 883, Mälardalens University, 72123 Västerås, Sweden:

${ }^{2}$ Unit for Preventive Nutrition, Department of Biosciences and Nutrition, Karolinska Institute, Huddinge, Sweden:

${ }^{3}$ Research Foundation - Flanders, Ghent, Belgium: ${ }^{4}$ Department of Public Health, Ghent University, Ghent, Belgium: ${ }^{5}$ Department of Nutrition and Food Science, University of the Basque Country, Vitoria, Spain: ${ }^{6}$ National Research Institute on Food and Nutrition, Rome, Italy: ${ }^{7}$ Department of Physiology, School of Medicine University of Granada, Granada, Spain: ${ }^{8}$ Department of Consumer \& Sensory Sciences, Campden BRI, UK: ${ }^{9}$ Grupo Inmunonutrición, Department Metabolismo y Nutrición, Madrid, Spain: ${ }^{10}$ Department of Nutrition and Dietetics, Harokopio University, Athens, Greece: " 1 Preventive Medicine and Nutrition Clinic, Department of Social Medicine, School of Medicine, University of Crete, Heraklion, Greece: ${ }^{12}$ Research Institute of Child Nutrition an der Rheinischen Friedrich-Wilhelms-Universität Bonn, Dortmund, Germany: ${ }^{13}$ Department of Pediatrics, Clinical Centre, University of Pécs, Pécs, Hungary: ${ }^{14}$ Institute of Environmental Medicine, Karolinska Institutet, Huddinge, Sweden: ${ }^{15}$ Division of Clinical Nutrition and Prevention, Department of Pediatrics, Medical University of Vienna, Vienna, Austria: ${ }^{16}$ Escuela Universitaria de Ciencias de la Salud, Universidad de Zaragoza, Zaragoza, Spain

Submitted 13 May 2011: Accepted 10 January 2012: First published online 21 February 2012

\begin{abstract}
Objective: To describe breakfast habits at food group level in European adolescents and to investigate the associations between these habits and sociodemographic factors. Design: Cross-sectional study.

Setting: Secondary schools from nine European cities participating in the HELENA (Healthy Lifestyle in Europe by Nutrition in Adolescence) Study. Breakfast habits were assessed twice using a computer-based $24 \mathrm{~h}$ dietary recall. Adolescents who consumed breakfast on at least one recall day were classified as 'breakfast consumers' and adolescents who did not have anything for breakfast on either of the two recall days were considered 'breakfast skippers'. A 'breakfast quality index' to describe breakfast quality was created based on the consumption or nonconsumption of cereals/cereal products, dairy products and fruits/vegetables. The sociodemographic factors studied were sex, age, region of Europe, maternal and paternal education, family structure and family affluence.

Subjects: Adolescents ( $n$ 2672, 53\% girls) aged 12-17 years.

Results: The majority of the adolescents reported a breakfast that scored poorly on the breakfast quality index. Older adolescents, adolescents from the southern part of Europe and adolescents from families with low socio-economic status were more likely to consume a low-quality breakfast.

Conclusions: The study highlights the need to promote the consumption of a highquality breakfast among adolescents, particularly in older adolescents, adolescents from southern Europe and adolescents from families with low socio-economic status, in order to improve public health.
\end{abstract}

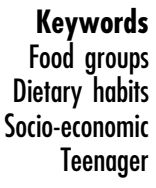

Breakfast consumption is an important part of a healthy lifestyle ${ }^{(1)}$. Regular breakfast consumption among adolescents has been associated with better diet quality ${ }^{(2-4)}$, better cognitive performance ${ }^{(5)}$ and reduced risk of becoming overweight or obese ${ }^{(6)}$. Nevertheless, breakfast skipping is a common behaviour among European adolescents, with a prevalence ranging from $3 \%{ }^{(7)}$ to $34 \%{ }^{(1)}$. 
A 'good-quality' breakfast can impact favourably on adolescent mental health ${ }^{(8)}$ and cognitive performance ${ }^{(9)}$ and improve the overall diet quality ${ }^{(3,10)}$. Preferably, breakfast should include products from each of the dairy, cereal and fruit groups ${ }^{(11)}$. However, these three food groups were found to be consumed for breakfast by only $10 \%$ of adolescents in studies from Belgium ${ }^{(3)}$ and The Netherlands ${ }^{(10)}$ and by $5 \%$ of adolescents in a study from Spain $^{(12)}$. Milk and cereal are commonly consumed at breakfast in many child and adolescent populations in Western countries $^{(1)}$, while fruit is less common ${ }^{(10,12)}$.

Previous studies in European adolescents have focused mainly on breakfast consumption $v$. skipping ${ }^{(6,13)}$. These studies have shown that daily breakfast consumption decreases with age ${ }^{(10,13,14)}$, and is associated with sex - girls were less likely to be daily breakfast consumers than boys $^{(10,13-16)}$ - and socio-economic factors ${ }^{(13,15,16)}$. Studies on breakfast quality and sociodemographic factors at European level among adolescents are, however, scarce. Low levels of breakfast consumption can be considered a public health issue but in order to be able to promote breakfast consumption it is first crucial to understand potential differences in breakfast habits among European adolescents, by sex, socio-economic factors and geographical factors.

The aim of the present study was therefore to describe the breakfast habits (in terms of both frequency and quality) at food group level in European adolescents. Additionally we wanted to investigate associations between these habits and sociodemographic factors such as sex, age, region in Europe, parental education, family structure and family affluence.

\section{Methods}

\section{Study design and sampling}

The HELENA (Healthy Lifestyle in Europe by Nutrition in Adolescence) Study is a school-based multi-centre study, designed to obtain reliable and valid data on nutrition and health-related factors from a sample of 3528 adolescents (52\% girls) aged $12 \cdot 50-17 \cdot 49$ years in ten European cities $^{(17)}$. The selected cities are Athens (Greece), Dortmund (Germany), Ghent (Belgium), Heraklion (Greece), Lille (France), Pecs (Hungary), Rome (Italy), Vienna (Austria), Stockholm/Västerås (Sweden) and Zaragoza (Spain).

The selection of the European cities was primarily a practical one. As it was not realistic to include a random sample of all European adolescents, it was decided to study a city-based sample, striving for representativeness of adolescents living in European cities. Within these cities, schools were randomly selected, but stratified for geographical location. Within the participating schools, classes were randomly selected, stratified by grade. In all countries it was compulsory to go to school at these ages and our aim was to reach a sub-sample from the whole population in these ages.
All pupils of the selected classes were invited to participate. A class was considered eligible if the participation rate was at least $70 \%$. The final database included only those participants who met the following criteria: they were between 12.50 and 17.49 years of age, they and their parents had provided written informed consent, and their weight and height had been measured. Participants were excluded if they were participating simultaneously in another clinical trial or had had an acute infection less than one week before the study. The adolescents completed in-class surveys, two non-consecutive computer-based $24 \mathrm{~h}$ dietary recalls and physical tests during the 2006-2007 academic year. A more detailed description of the study design, sampling and procedure has previously been published $^{(17)}$. Both parents and adolescents gave written informed consent, and the HELENA study protocol was approved by the national or local independent ethical committee from the relevant European city ${ }^{(18)}$.

The present paper includes data of 2672 adolescents from nine cities ( $53 \%$ girls), with a mean age of 14.8 (SD $1 \cdot 2$ ) years. Data from Heraklion (Greece) are not used in the present analysis because only one $24 \mathrm{~h}$ dietary recall was collected in the majority of adolescents.

\section{Sociodemographic measurements}

Socio-economic factors were assessed, during the in-class surveys, via the questionnaire 'Your Living Environment ${ }^{(19)}$. This questionnaire gathered general information about the education level of parents, family structure and family affluence. This latter variable was measured using a modified version of the family affluence scale (FAS), a scale developed by the WHO collaborative Health Behaviour in School-aged Children (HBSC) Study ${ }^{(20)}$. A sum score of the following items was used: whether the adolescent had his/her own bedroom, the number of cars in the family, the number of computers and the presence of an Internet connection at home. The FAS was dichotomized to 'low family affluence' (FAS score $\leq 3$ ) $v$. 'high family affluence' (FAS score $\geq 4$ ).

Maternal and paternal education level was dichotomized into 'low/medium education' (elementary, lower secondary, higher secondary education) and 'high education' (university education). The family structure variable was derived from the question 'With whom do you principally stay?'. Adolescents who chose 'with both of your parents', 'with your mother and her partner' or 'with your father and his partner' were considered to have a 'traditional' family structure. Adolescents who chose 'with your mother', 'with your father', 'with your mother half of the time and your father half of the time', 'with your grandparents or other relatives', 'with your foster or adoptive parents' or 'in an orphanage or somewhere else' were considered to have a 'single-parent/shared-care' family structure.

Data on sex, age and study centre were collected by a physician. Age was dichotomized to younger or older than 15 years of age. The variable 'study centre' was 
dichotomized into northern/central (Dortmund, Ghent, Lille, Pecs, Vienna and Stockholm/Västerås) and southern (Athens, Rome and Zaragoza).

All of the questionnaires included in the study were discussed and approved by the HELENA partners. After consensus was reached, the questionnaires were translated and back-translated into the native language of the participants $^{(19)}$.

\section{Dietary survey}

Dietary intake data were obtained using a dietary assessment tool named HELENA-DIAT, which was based on a self-administered, computerized $24 \mathrm{~h}$ dietary recall program called Young Adolescents' Nutrition Assessment on Computer (YANA-C) ${ }^{(21,22)}$. The program contains questions related to six meal occasions (i.e. breakfast, morning snacks, lunch, afternoon snacks, evening meal, evening snacks) with questions designed to prompt the adolescents to remember what they ate. The first two questions asked about the time they got up the previous day and if they had breakfast. If they responded no, they were prompted with an additional question 'You didn't have anything, however small, to eat or drink for breakfast?'. If the adolescents had breakfast, a drink or something small, they were asked 'Where and with whom did you have breakfast, yesterday? and 'Around what time was that?'. Then, adolescents selected the food items consumed from a culturally adapted list and further described the quantity consumed. Pictures and measurement units helped the adolescents to give quantitatively detailed information ${ }^{(21,22)}$. Two computerized $24 \mathrm{~h}$ recalls were performed on two nonconsecutive days within the space of two weeks. The HELENA-DIAT was filled in by the adolescents at school, and it took about $10-30 \mathrm{~min}$ to complete.

\section{Breakfast patterns}

Based on reported breakfast consumption the adolescent was classified as a 'breakfast consumer' (i.e. they consumed breakfast on at least one recall day) or a 'breakfast skipper' (i.e. they did not consume breakfast on either of the two recall days). According to one previous study ${ }^{(23)}$, a breakfast meal should weigh more than $15 \mathrm{~g}$ to be considered a meal. In our study five breakfast meals weighed less than $15 \mathrm{~g}$ and were excluded. The computer program initially categorized foods into twenty-seven food groups (Table 1), but because many of the food groups were infrequently consumed for breakfast we merged similar groups and reduced this number to seventeen (Tables 2 to 4). All food items were classified as being consumed (on one or both of the recall days) or not consumed (not on either of the two recall days). For the purpose of the study, breakfast quality was related to the presence (irrespective of amount) of food items from three specific food groups: cereal products ('bread' and 'breakfast cereals'), dairy products ('milk and yoghurt' and 'cheese') and fruit/vegetables ('fruit \& vegetables' and 'fruit juice'). Additionally, a 'breakfast quality index' was created in which 1 point was awarded for consuming a breakfast and an extra 1 point was awarded for each of the three food groups (hereafter called 'target food groups') consumed (i.e. 1 to 3 extra points), resulting in a possible score of 0 to 8 across both days. Adolescents consuming a breakfast that scored highly ( $\geq 6$ points) were considered to have a 'high quality breakfast' while those who scored lowly ( $<4$ points) were considered to have a 'low quality breakfast'.

\section{Statistical analyses}

All analyses were performed using the statistical software package IBM SPSS Statistics version $19 \cdot 0$ for Windows (IBM SPSS Inc., Chicago, IL, USA), and the level of significance was set at $5 \%$. We used percentage, median and 25th and 75th percentiles for describing breakfast consumers and the total quantity of each food group consumed for breakfast $(\mathrm{g} / \mathrm{d})$.

Multiple logistic regression analyses were used to investigate the associations with breakfast consumption and food groups for breakfast (dependent variables) according to sociodemographic variables (independent variables). All multiple logistic regression models included sex (boy $v$. girl), age ( $<15$ years $v . \geq 15$ years), region (southern $v$. northern/central), maternal and paternal education level (low/medium $v$. high), family structure (traditional $v$. single parent/shared care) and FAS score (low $v$. high) as independent variables.

Multiple linear regression analyses were performed to evaluate associations between the breakfast quality index (continuous) and sex, age (in years, continuous), region, maternal and paternal education level, family structure and FAS score as independent variables. All analyses were adjusted by a weighting factor to balance the sample according to the age and sex distribution of the theoretical sample, to guarantee representation of each of the stratified groups.

\section{Results}

\section{Population characteristics}

Table 2 shows that $53 \%$ of the population were girls, $56 \%$ were under 15 years of age and $29 \%$ came from the southern part of Europe. One-third of the adolescents had highly educated mothers, one-third had highly educated fathers and $80 \%$ were living in a traditional family structure. Three-quarters (77\%) indicated that their family was affluent, as defined by the FAS.

\section{Breakfast consumption according to sociodemographic factors}

We observed that $7 \%$ of the adolescents were breakfast skippers' (Fig. 1). Breakfast consumption, on both recall days, was reported among $77 \%$ of the adolescents (data not shown). Younger adolescents $(<15$ years of age) reported more often than older adolescents that they 
Table 1 Percentage of adolescents consuming twenty-seven food groups and the median (P25-P75) consumption on the days the food was consumed (observed sample): adolescents ( $n$ 2672) aged 12-17 years from nine European cities participating in the HELENA (Healthy Lifestyle in Europe by Nutrition in Adolescence) Study

\begin{tabular}{|c|c|c|c|c|}
\hline \multirow[b]{2}{*}{ Food group } & \multicolumn{2}{|c|}{$\%$ of consumers } & \multicolumn{2}{|c|}{ g consumed $^{*}$} \\
\hline & One day only & Both days & Median & P25-P75 \\
\hline \multicolumn{5}{|l|}{ Beverages } \\
\hline Tea \& coffee & $11 \cdot 0$ & $8 \cdot 8$ & 214 & $112-225$ \\
\hline Water & $16 \cdot 0$ & $7 \cdot 3$ & 200 & $175-275$ \\
\hline Fruit juice & $15 \cdot 0$ & $9 \cdot 7$ & 200 & $200-265$ \\
\hline Soft drinks & $5 \cdot 9$ & $1 \cdot 4$ & 250 & $200-425$ \\
\hline \multicolumn{5}{|l|}{ Dairy } \\
\hline Milk & $23 \cdot 0$ & $28 \cdot 0$ & 225 & $150-262$ \\
\hline Soya drink & $0 \cdot 1$ & 0.3 & 200 & $148-244$ \\
\hline Sugared milk & $11 \cdot 0$ & $5 \cdot 2$ & 225 & $200-236$ \\
\hline Yoghurt & $5 \cdot 7$ & $1 \cdot 7$ & 169 & $125-225$ \\
\hline Cheese & $13 \cdot 0$ & $4 \cdot 8$ & 30 & $20-44$ \\
\hline Other milk products† & $1 \cdot 0$ & $0 \cdot 1$ & 62 & $7 \cdot 5-200$ \\
\hline \multicolumn{5}{|c|}{ Meat, fish, eggs and their products } \\
\hline Meat \& products & $14 \cdot 0$ & $5 \cdot 1$ & 33 & $22 \cdot 5-50$ \\
\hline Fish \& products & $0 \cdot 6$ & $0 \cdot 1$ & 21 & 14-33 \\
\hline Eggs & $3 \cdot 6$ & 0.4 & 50 & $50-100$ \\
\hline \multicolumn{5}{|l|}{ Fruit \& vegetables } \\
\hline Fruits & $9 \cdot 7$ & $3 \cdot 7$ & 130 & $100-184$ \\
\hline Vegetables & $5 \cdot 5$ & $1 \cdot 1$ & 30 & $10-61$ \\
\hline Other vegetables $\ddagger$ & 1.5 & $0 \cdot 1$ & 38 & $20-88$ \\
\hline \multicolumn{5}{|l|}{ Cereals } \\
\hline Bread & $26 \cdot 0$ & $22 \cdot 0$ & 60 & $36-80$ \\
\hline Breakfast cereals & $14 \cdot 0$ & $9 \cdot 3$ & 40 & $28-58$ \\
\hline Other cereals§ & 0.4 & 0 & 68 & $45-73$ \\
\hline \multicolumn{5}{|l|}{ Cakes \& sweets } \\
\hline Cakes/pies/biscuits & $19 \cdot 0$ & $8 \cdot 8$ & 68 & $48-100$ \\
\hline Snacks & 0.7 & $0 \cdot 1$ & 55 & $30-80$ \\
\hline Added sugar & $13 \cdot 0$ & $6 \cdot 8$ & 20 & $12-38$ \\
\hline Sweets & $14 \cdot 0$ & $8 \cdot 0$ & 30 & $15-60$ \\
\hline \multicolumn{5}{|l|}{ Added fat/oil } \\
\hline Vegetable fat & $8 \cdot 4$ & $3 \cdot 3$ & 16 & $8 \cdot 1-24$ \\
\hline Butter & $11 \cdot 0$ & $5 \cdot 9$ & 15 & $9 \cdot 0-24$ \\
\hline \multicolumn{5}{|l|}{ Miscellaneous } \\
\hline Pasta, soup, etc. & $2 \cdot 0$ & $0 \cdot 1$ & 60 & $2 \cdot 7-150$ \\
\hline Sauce & $1 \cdot 9$ & 0.2 & 41 & $20-74$ \\
\hline
\end{tabular}

P25, 25th percentile; $P 75,75$ th percentile.

*Gram consumed on consumption days.

tMilk-based dessert and cream.

‡Potatoes, pulses, etc.

$\S$ Oats and rice.

were 'breakfast consumers' $(\mathrm{OR}=1 \cdot 66 ; 95 \%$ CI $1 \cdot 17$, 2.35). Fewer adolescents with a low/medium maternal education level indicated that they were 'breakfast consumers' compared with adolescents with a high maternal education level (OR $=0.61 ; 95 \%$ CI $0 \cdot 38,0 \cdot 98)$. There were no significant differences in breakfast consumption ('consumer' $v$. 'skipper') by sex, region, paternal education, family structure and FAS (Tables 3 and 4).

\section{Breakfast quality according to sociodemographic factors}

We found that $4 \%$ of the sample consumed all three of the target food groups on both recall days (Fig. 1). Figure 1 also shows that $53 \%$ of the adolescents consumed products from two or fewer of the target food groups.

Breakfast quality was higher in adolescents from the northern and central part of Europe $(\beta=0 \cdot 142, P<0 \cdot 001)$, in those whose mothers had a high education level $(\beta=0 \cdot 129, P<0 \cdot 001)$, in those reporting higher family affluence $(\beta=0.068, P=0.002)$ and in those coming from a traditional family structure $(\beta=0 \cdot 055, P=0 \cdot 006)$. Breakfast quality was lower among older adolescents than among younger $(\beta=-0 \cdot 063, P=0 \cdot 001)$. There was no association between breakfast quality and sex or paternal education (Table 5).

\section{Food groups consumed at breakfast according to sociodemographic factors}

Table 3 shows that girls were more likely to consume 'tea and coffee' and 'butter' but less likely to consume 'meat, fish and eggs' (all $P<0 \cdot 05$ ) for breakfast than boys. Younger adolescents ( $<15$ years of age) were more likely to consume 'other milk products' $(P<0 \cdot 001)$ and less likely to consume 'tea and coffee', 'water' and 'added sugar' (all $P<0 \cdot 05$ ) than older adolescents. Adolescents from northern/central parts of Europe were more likely to 
Table 2 Percentage of consumers of breakfast and of seventeen food groups for breakfast by sociodemographic factors (observed sample): adolescents ( $n$ 2672) aged $12-17$ years from nine European cities participating in the HELENA (Healthy Lifestyle in Europe by Nutrition in Adolescence) Study

\begin{tabular}{|c|c|c|c|c|c|c|c|c|c|c|c|c|c|c|}
\hline & \multicolumn{2}{|c|}{$\operatorname{Sex}^{*}$} & \multicolumn{2}{|c|}{ Age $^{*}$ (years) } & \multicolumn{2}{|c|}{ Region* } & \multicolumn{2}{|c|}{ Maternal education* } & \multicolumn{2}{|c|}{ Paternal education* } & \multicolumn{2}{|c|}{ Family structure* } & \multicolumn{2}{|c|}{ FAS* } \\
\hline & Boy & Girl & $<15$ & $\geq 15$ & North/central & South & Low/medium & High & Low/medium & High & $\begin{array}{l}\text { Single parent/ } \\
\text { shared care }\end{array}$ & Traditional & Low & High \\
\hline n 2672 & 1245 & 1427 & 1496 & 1179 & 1904 & 768 & 1642 & 901 & 1573 & 885 & 530 & 2069 & 559 & 1880 \\
\hline$\% 100$ & 47 & 53 & 56 & 44 & 71 & 29 & 65 & 35 & 64 & 36 & 20 & 80 & 23 & 77 \\
\hline Breakfast consumert & 93 & 93 & 95 & 91 & 93 & 92 & 92 & 96 & 92 & 96 & 92 & 93 & 91 & 94 \\
\hline \multicolumn{15}{|l|}{ Beverages } \\
\hline Tea \& coffee & 18 & 22 & 17 & 23 & 21 & 16 & 21 & 17 & 21 & 18 & 18 & 21 & 21 & 19 \\
\hline Water & 22 & 24 & 21 & 25 & 24 & 20 & 24 & 21 & 23 & 22 & 24 & 21 & 22 & 23 \\
\hline Fruit juice & 24 & 26 & 25 & 25 & 29 & 16 & 20 & 32 & 22 & 30 & 26 & 22 & 17 & 28 \\
\hline Soft drinks & 8 & 6 & 8 & 7 & 10 & 1 & 10 & 2 & 9 & 5 & 7 & 9 & 7 & 7 \\
\hline \multicolumn{15}{|l|}{ Dairy } \\
\hline Milk \& yoghurt & 58 & 52 & 57 & 52 & 49 & 50 & 50 & 64 & 51 & 63 & 55 & 53 & 50 & 56 \\
\hline Cheese & 19 & 18 & 18 & 19 & 23 & 6 & 16 & 20 & 18 & 18 & 18 & 19 & 17 & 19 \\
\hline Other milk products $\ddagger$ & 17 & 17 & 20 & 14 & 19 & 11 & 17 & 18 & 17 & 18 & 17 & 18 & 19 & 17 \\
\hline \multicolumn{15}{|l|}{ Meat, fish, eggs \& their products } \\
\hline Meat, fish \& eggs & 24 & 20 & 21 & 23 & 28 & 7 & 23 & 20 & 24 & 19 & 21 & 25 & 24 & 22 \\
\hline \multicolumn{15}{|l|}{ Fruit \& vegetables } \\
\hline Fruit \& Vegetables & 19 & 21 & 20 & 19 & 25 & 8 & 18 & 24 & 19 & 23 & 21 & 18 & 16 & 20 \\
\hline \multicolumn{15}{|l|}{ Cereals } \\
\hline Bread & 46 & 49 & 56 & 48 & 58 & 20 & 45 & 52 & 44 & 50 & 48 & 47 & 41 & 49 \\
\hline Cereals§ & 27 & 24 & 25 & 24 & 26 & 21 & 21 & 30 & 21 & 30 & 26 & 23 & 16 & 28 \\
\hline \multicolumn{15}{|l|}{ Cakes \& sweets } \\
\hline Cakes, pies, biscuits \& snacks & 28 & 30 & 30 & 26 & 23 & 41 & 29 & 29 & 28 & 30 & 29 & 27 & 32 & 28 \\
\hline Added sugar & 21 & 20 & 18 & 22 & 18 & 26 & 21 & 20 & 21 & 21 & 21 & 19 & 20 & 22 \\
\hline Sweets including chocolate & 22 & 23 & 23 & 22 & 21 & 27 & 22 & 24 & 21 & 25 & 24 & 16 & 17 & 24 \\
\hline \multicolumn{15}{|l|}{ Added fat/oil } \\
\hline Vegetable fat & 11 & 12 & 11 & 13 & 15 & 3 & 11 & 12 & 12 & 12 & 12 & 10 & 14 & 11 \\
\hline Butter & 16 & 18 & 17 & 17 & 22 & 4 & 15 & 20 & 15 & 19 & 17 & 19 & 10 & 19 \\
\hline \multicolumn{15}{|l|}{ Miscellaneous } \\
\hline Pasta, soup, sauce, etc. & 4 & 3 & 2 & 2 & 2 & 1 & 2 & 1 & 2 & 1 & 2 & 2 & 2 & 2 \\
\hline
\end{tabular}

FAS, family affluence

The proportion of adolescents who consumed the different food items (on one or both days).

tBreakfast consumption on at least one day.

\$Breakfast cereals and other cereals. 
Table 3 Results of multivariate logistic regression analyses with consumption of breakfast and of food groups as dependent variables and sociodemographic factors as independent variables: adolescents $(n 2672)$ aged $12-17$ years from nine European cities participating in the HELENA (Healthy Lifestyle in Europe by Nutrition in Adolescence) Study

\begin{tabular}{|c|c|c|c|c|c|c|c|c|c|}
\hline & \multicolumn{3}{|c|}{ Girls } & \multicolumn{3}{|c|}{$<15$ years of age } & \multicolumn{3}{|c|}{ Northern/central Europe } \\
\hline & OR & $95 \% \mathrm{Cl}$ & $P$ & OR & $95 \% \mathrm{Cl}$ & $P$ & OR & $95 \% \mathrm{Cl}$ & $P$ \\
\hline $\begin{array}{l}\text { Breakfast consumer* } \\
\text { Beverages }\end{array}$ & 1.09 & $0 \cdot 78,1 \cdot 53$ & $0 \cdot 613$ & $1 \cdot 66$ & $1 \cdot 17,2 \cdot 35$ & 0.005 & $1 \cdot 08$ & $0 \cdot 74,1.57$ & $0 \cdot 707$ \\
\hline Tea \& coffee & $1 \cdot 33$ & $1 \cdot 07,1 \cdot 64$ & 0.009 & 0.57 & $0.46,0.70$ & $<0.001$ & 1.45 & $1 \cdot 13,1 \cdot 87$ & 0.004 \\
\hline Water & $1 \cdot 16$ & $0.96,1.42$ & $0 \cdot 133$ & $0 \cdot 81$ & $0.66,0.99$ & 0.037 & $1 \cdot 25$ & $0.99,1.57$ & 0.060 \\
\hline Fruit juice & $1 \cdot 17$ & $0.96,1.43$ & $0 \cdot 116$ & $1 \cdot 10$ & $0.90,1.34$ & 0.363 & $1 \cdot 85$ & $1 \cdot 45,2 \cdot 36$ & $<0.001$ \\
\hline Soft drinks & $0 \cdot 77$ & $0.55,1.08$ & $0 \cdot 125$ & $1 \cdot 32$ & $0.94,1.84$ & $0 \cdot 109$ & $8 \cdot 75$ & $4 \cdot 16,18 \cdot 39$ & $<0.001$ \\
\hline \multicolumn{10}{|l|}{ Dairy } \\
\hline Milk \& yoghurt & $0 \cdot 87$ & $0 \cdot 73,1 \cdot 03$ & $0 \cdot 111$ & $1 \cdot 15$ & $0 \cdot 97,1 \cdot 37$ & $0 \cdot 113$ & $0 \cdot 34$ & $0.28,0.42$ & $<0.001$ \\
\hline Cheese & $1 \cdot 05$ & $0 \cdot 84,1 \cdot 31$ & 0.674 & $1 \cdot 07$ & $0.85,1.33$ & 0.567 & $4 \cdot 50$ & $3 \cdot 18,6 \cdot 38$ & $<0.001$ \\
\hline Other milk productst & $1 \cdot 00$ & $0 \cdot 80,1 \cdot 26$ & 0.973 & $1 \cdot 72$ & $1 \cdot 37,2 \cdot 17$ & $<0.001$ & $2 \cdot 34$ & $1 \cdot 74,3 \cdot 14$ & $<0.001$ \\
\hline \multicolumn{10}{|c|}{ Meat, fish, eggs \& their products } \\
\hline Meat, fish \& eggs & 0.79 & $0.64,0.98$ & $0 \cdot 028$ & 0.96 & $0 \cdot 78,1 \cdot 18$ & 0.678 & $5 \cdot 77$ & $4 \cdot 12,8 \cdot 08$ & $<0.001$ \\
\hline \multicolumn{10}{|l|}{ Fruit \& vegetables } \\
\hline Fruit \& vegetables & $1 \cdot 22$ & $0 \cdot 98,1 \cdot 51$ & 0.072 & $1 \cdot 11$ & $0 \cdot 90,1 \cdot 38$ & 0.331 & $4 \cdot 39$ & $3 \cdot 17,6 \cdot 08$ & $<0.001$ \\
\hline \multicolumn{10}{|l|}{ Cereals } \\
\hline Bread & $1 \cdot 19$ & $0.99,1.42$ & 0.061 & $1 \cdot 06$ & $0 \cdot 88,1 \cdot 26$ & 0.550 & $5 \cdot 92$ & $4 \cdot 71,7 \cdot 44$ & $<0.001$ \\
\hline Cereals $\ddagger$ & $0 \cdot 89$ & $0 \cdot 73,1 \cdot 08$ & $0 \cdot 240$ & $1 \cdot 15$ & $0.94,1.39$ & $0 \cdot 173$ & $1 \cdot 23$ & $0.98,1.54$ & 0.080 \\
\hline \multicolumn{10}{|l|}{ Cakes and sweets } \\
\hline $\begin{array}{l}\text { Cakes, pies, biscuits \& } \\
\text { snacks }\end{array}$ & $1 \cdot 06$ & $0 \cdot 88,1 \cdot 28$ & 0.517 & $1 \cdot 04$ & $0 \cdot 86,1 \cdot 25$ & 0.695 & $0 \cdot 42$ & $0.35,0.52$ & $<0.001$ \\
\hline Added sugar & $1 \cdot 06$ & $0 \cdot 87,1 \cdot 30$ & 0.564 & $0 \cdot 70$ & $0.57,0.86$ & 0.001 & $0 \cdot 61$ & $0 \cdot 49,0.77$ & $<0.001$ \\
\hline Sweets including chocolate & $1 \cdot 02$ & $0 \cdot 84,1 \cdot 25$ & $0 \cdot 816$ & 0.94 & $0 \cdot 77,1 \cdot 15$ & 0.569 & 0.63 & $0.50,0.78$ & $<0.001$ \\
\hline \multicolumn{10}{|l|}{ Added fat/oil } \\
\hline Vegetable fat & 1.06 & $0 \cdot 81,1 \cdot 37$ & 0.685 & 0.99 & $0 \cdot 76,1 \cdot 29$ & 0.929 & $7 \cdot 64$ & $4 \cdot 63,12 \cdot 61$ & $<0.001$ \\
\hline Butter & $1 \cdot 35$ & $1 \cdot 07,1 \cdot 70$ & 0.013 & $1 \cdot 07$ & $0 \cdot 85,1 \cdot 35$ & 0.561 & $8 \cdot 47$ & $5 \cdot 28,13 \cdot 59$ & $<0.001$ \\
\hline \multicolumn{10}{|l|}{ Miscellaneous } \\
\hline Pasta, soup, sauce, etc. & $0 \cdot 80$ & $0 \cdot 52,1 \cdot 21$ & 0.289 & 0.68 & $0 \cdot 44,1 \cdot 05$ & 0.082 & $3 \cdot 30$ & $1 \cdot 72,6 \cdot 36$ & $<0.001$ \\
\hline
\end{tabular}

Odds ratios and $95 \%$ confidence intervals weighted for age and sex. Reference categories: boys, $\geq 15$ years of age and southern Europe.

*Breakfast consumed on one or both of the recall days.

tMilk-based dessert, sugared milk and cream.

$\ddagger$ Breakfast cereals and other cereals.

consume a drink (tea, coffee, fruit juice and soft drink; all $P<0 \cdot 001$ ) for breakfast compared with those from the southern part of Europe. They were also more likely to consume 'cheese', 'other milk products', 'meat, fish and eggs', 'fruit and vegetables', 'bread', 'cereals' and 'added fat or oil' and less likely to consume 'milk and yoghurt', 'cakes', 'sweets' and 'added sugar' (all $P<0.001$ ) for breakfast compared with their counterparts from southern Europe.

Adolescents whose mothers had a low/medium education level were less likely to consume 'fruit juice' and 'milk and yoghurt' and more likely to consume 'soft drinks' (all $P<0 \cdot 001$ ) and 'fruit and vegetables' and 'bread' (both $P<0 \cdot 05$ ) for breakfast compared with those whose mothers had a high education level (Table 4). Adolescents from single-parent/shared-care families were less likely to consume 'sweets' $(P<0 \cdot 01)$ for breakfast compared with those from traditional families. Adolescents who reported lower family affluence were less likely to consume 'fruit juice' and 'milk and yoghurt' (both $P<0.01$ ) and to consume 'cereals' and 'sweets' (both $P<0 \cdot 001$ ); they were also more likely to consume 'other milk products' $(P<0 \cdot 01)$, 'meat, fish and eggs' and 'vegetable fat' (both $P<0.001$ ) for breakfast compared with those who reported greater family affluence. There were no significant differences in food groups consumed for breakfast by level of paternal education (Table 4).

\section{Discussion}

The aim of the present study was to describe breakfast habits (in terms of both frequency and quality) at food group level in European adolescents. Additionally we wanted to investigate the associations between these habits and sociodemographic factors such as sex, age, region in Europe, parental education, family structure and family affluence.

The main findings of the study are that the majority of the adolescents studied consumed some form of breakfast, but that the quality was rather low. We also found that breakfast habits were associated with age, region in Europe, maternal education, family structure and family affluence. Breakfast skipping (not consuming breakfast on any of the two recall days) was reported by $7 \%$ of the adolescents, a figure which is in agreement with other studies among European adolescents ${ }^{(1,13)}$. With regard to breakfast quality, our study shows that the majority of the 
Table 4 Results of multivariate logistic regression analyses with consumption of breakfast and of food groups as dependent variables and sociodemographic factors as independent variables: adolescents ( $n$ 2672) aged 12-17 years from nine European cities participating in the HELENA (Healthy Lifestyle in Europe by Nutrition in Adolescence) Study

\begin{tabular}{|c|c|c|c|c|c|c|c|c|c|c|c|c|}
\hline & \multicolumn{3}{|c|}{ Low/medium maternal education } & \multicolumn{3}{|c|}{ Low/medium paternal education } & \multicolumn{3}{|c|}{$\begin{array}{l}\text { Single-parent/shared-care } \\
\text { family structure }\end{array}$} & \multicolumn{3}{|c|}{ Low FAS } \\
\hline & OR & $95 \% \mathrm{Cl}$ & $P$ & OR & $95 \% \mathrm{Cl}$ & $P$ & OR & $95 \% \mathrm{Cl}$ & $P$ & OR & $95 \% \mathrm{Cl}$ & $P$ \\
\hline $\begin{array}{l}\text { Breakfast consumer* } \\
\text { Beverages }\end{array}$ & $0 \cdot 61$ & $0.38,0.98$ & 0.040 & $0 \cdot 80$ & $0 \cdot 51,1 \cdot 27$ & $0 \cdot 351$ & $1 \cdot 04$ & $0 \cdot 67,1 \cdot 60$ & $0 \cdot 874$ & $0 \cdot 73$ & $0.49,1.08$ & $0 \cdot 118$ \\
\hline Tea \& coffee & 1.09 & $0.83,1 \cdot 42$ & 0.548 & 1.09 & $0.83,1.43$ & 0.533 & $1 \cdot 10$ & $0.84,1 \cdot 42$ & 0.490 & $1 \cdot 14$ & $0 \cdot 88,1 \cdot 48$ & 0.328 \\
\hline Water & 1.05 & $0.82,1.35$ & 0.713 & $1 \cdot 02$ & $0.79,1.31$ & 0.895 & $0 \cdot 86$ & $0 \cdot 67,1 \cdot 12$ & 0.269 & 1.08 & $0.84,1.38$ & 0.566 \\
\hline Fruit juice & 0.63 & $0 \cdot 49,0.80$ & $<0.001$ & $0 \cdot 88$ & $0 \cdot 69,1 \cdot 12$ & 0.292 & $0 \cdot 81$ & $0 \cdot 62,1 \cdot 05$ & $0 \cdot 107$ & $0 \cdot 64$ & $0.48,0.85$ & 0.002 \\
\hline Soft drinks & $3 \cdot 83$ & $2 \cdot 23,6 \cdot 56$ & $<0.001$ & 1.09 & $0 \cdot 69,1 \cdot 74$ & $0 \cdot 71$ & $1 \cdot 18$ & $0 \cdot 79,1 \cdot 76$ & 0.408 & 0.92 & $0 \cdot 60,1 \cdot 40$ & 0.687 \\
\hline \multicolumn{13}{|l|}{ Dairy } \\
\hline Milk \& yoghurt & 0.62 & $0 \cdot 50,0.77$ & $<0.001$ & $0 \cdot 81$ & $0 \cdot 65,1 \cdot 02$ & 0.067 & $1 \cdot 05$ & $0 \cdot 84,1 \cdot 31$ & 0.661 & $0 \cdot 70$ & $0.56,0.88$ & 0.002 \\
\hline Cheese & $0 \cdot 78$ & $0.59,1.03$ & 0.084 & 1.03 & $0 \cdot 78,1 \cdot 37$ & 0.835 & $1 \cdot 01$ & $0.76,1 \cdot 33$ & 0.960 & $1 \cdot 29$ & $0.97,1.73$ & 0.081 \\
\hline Other milk productst & 1.04 & $0 \cdot 78,1.39$ & $0 \cdot 797$ & $0 \cdot 80$ & $0 \cdot 60,1.07$ & $0 \cdot 126$ & $1 \cdot 02$ & $0 \cdot 77,1 \cdot 36$ & 0.892 & 1.50 & $1 \cdot 23,1.98$ & 0.005 \\
\hline \multicolumn{13}{|l|}{ Meat, fish, eggs \& their products } \\
\hline Meat, fish \& eggs & 0.93 & $0 \cdot 71,1 \cdot 22$ & 0.593 & $1 \cdot 27$ & $0.96,1.67$ & 0.090 & $1 \cdot 04$ & $0 \cdot 81,1 \cdot 35$ & $0 \cdot 749$ & $1 \cdot 58$ & $1 \cdot 21,2 \cdot 06$ & 0.001 \\
\hline \multicolumn{13}{|l|}{ Fruit \& vegetables } \\
\hline Fruit \& vegetables & $0 \cdot 75$ & $0.57,0.99$ & 0.039 & $0 \cdot 91$ & $0 \cdot 69,1 \cdot 20$ & 0.503 & $0 \cdot 76$ & $0.57,1.01$ & 0.057 & $1 \cdot 13$ & $0 \cdot 85,1 \cdot 51$ & 0.396 \\
\hline \multicolumn{13}{|l|}{ Cereals } \\
\hline Bread & $0 \cdot 79$ & $0.63,0.99$ & 0.043 & $0 \cdot 83$ & $0.66,1 \cdot 04$ & $0 \cdot 104$ & $0 \cdot 86$ & $0.69,1.08$ & $0 \cdot 208$ & $1 \cdot 15$ & $0.91,1.45$ & 0.232 \\
\hline Cereals & $0 \cdot 85$ & $0 \cdot 67,1 \cdot 08$ & $0 \cdot 183$ & $0 \cdot 76$ & $0.60,0.96$ & 0.024 & 0.94 & $0 \cdot 73,1 \cdot 21$ & 0.615 & 0.54 & $0.41,0.72$ & $<0.001$ \\
\hline \multicolumn{13}{|l|}{ Cakes and sweets } \\
\hline $\begin{array}{l}\text { Cakes, pies, biscuits \& } \\
\text { snacks }\end{array}$ & 0.98 & $0 \cdot 77,1 \cdot 24$ & 0.867 & $0 \cdot 89$ & $0 \cdot 70,1 \cdot 13$ & 0.342 & $1 \cdot 00$ & $0 \cdot 79,1 \cdot 28$ & 0.970 & $1 \cdot 02$ & $0 \cdot 80,1 \cdot 29$ & 0.900 \\
\hline Added sugar & $1 \cdot 05$ & $0 \cdot 81,1 \cdot 36$ & $0 \cdot 711$ & 0.94 & $0 \cdot 73,1 \cdot 21$ & 0.634 & $0 \cdot 84$ & $0 \cdot 64,1 \cdot 10$ & $0 \cdot 209$ & $0 \cdot 84$ & $0 \cdot 65,1 \cdot 10$ & $0 \cdot 210$ \\
\hline Sweets including chocolate & $1 \cdot 10$ & $0.85,1 \cdot 41$ & 0.468 & $0 \cdot 72$ & $0.56,0.93$ & 0.010 & $0 \cdot 68$ & $0.52,0.90$ & 0.007 & 0.57 & $0.43,0.76$ & $<0.001$ \\
\hline \multicolumn{13}{|l|}{ Added fat/oil } \\
\hline Vegetable fat & $1 \cdot 13$ & $0 \cdot 80,1 \cdot 60$ & 0.495 & $0 \cdot 77$ & $0.55,1.09$ & $0 \cdot 138$ & 0.73 & $0.51,1.03$ & 0.077 & $2 \cdot 12$ & $1 \cdot 54,2 \cdot 92$ & $<0.001$ \\
\hline Butter & $0 \cdot 78$ & $0.58,1.04$ & 0.092 & $0 \cdot 83$ & $0 \cdot 62,1 \cdot 11$ & $0 \cdot 214$ & $1 \cdot 11$ & $0.84,1.49$ & 0.454 & $0 \cdot 73$ & $0.52,1.03$ & 0.075 \\
\hline \multicolumn{13}{|l|}{ Miscellaneous } \\
\hline Pasta, soup, sauce, etc. & $1 \cdot 20$ & $0 \cdot 68,2 \cdot 12$ & 0.533 & $1 \cdot 35$ & $0 \cdot 76,2 \cdot 40$ & 0.314 & $0 \cdot 80$ & $0.46,1.39$ & 0.426 & 1.55 & $0.94,2.56$ & 0.083 \\
\hline
\end{tabular}

FAS, family affluence scale.

FAS,

Bilkas cons.

fBreakfast cereals and other cereals. 


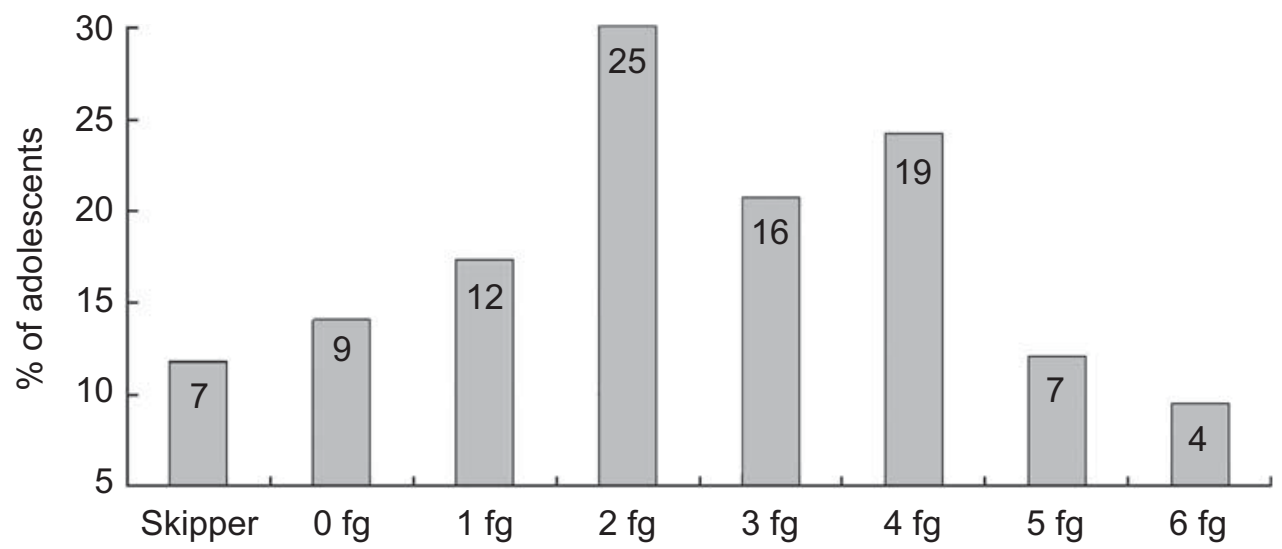

Fig. 1 Breakfast skippers and total number of target food groups ( $\mathrm{fg}$ ) consumed at breakfast over both recall days (maximum: three per day) in the study population: adolescents ( $n$ 2672) aged 12-17 years from nine European cities participating in the HELENA (Healthy Lifestyle in Europe by Nutrition in Adolescence) Study

Table 5 Multiple regression analyses exploring the association between breakfast quality index ${ }^{*}$ and sociodemographic factors: adolescents ( $n$ 2672) aged 12-17 years from nine European cities participating in the HELENA (Healthy Lifestyle in Europe by Nutrition in Adolescence) Study

\begin{tabular}{lrr}
\hline & \multicolumn{1}{c}{$\beta$} & \multicolumn{1}{c}{$P$} \\
\hline Girls & 0.012 & 0.536 \\
Age (years) $\dagger$ & -0.063 & 0.001 \\
Northern/central Europe & 0.142 & $<0.001$ \\
High maternal education & 0.129 & $<0.001$ \\
High paternal education & 0.031 & 0.208 \\
Traditional family structure & 0.055 & 0.006 \\
High FAS & 0.068 & 0.002
\end{tabular}

FAS, family affluence scale.

*Breakfast quality index score has nine categories from skipper (score of 0 ) to three target food groups consumed on both days (score of 8 ). tAge is a continuous variable.

adolescents (96\%) did not consume a breakfast including foods from all three of the target food groups on both recall days. Additionally, half of the adolescents consumed products from no more than two of the target food groups together during both the recall days. Similar results were reported in a Dutch study ${ }^{(10)}$ and in Spanish children and adolescents ${ }^{(12)}$.

Sex differences in breakfast consumption have been reported in other studies ${ }^{(1,13)}$; however, we found no differences between boys and girls in either frequency of breakfast consumption or breakfast quality. We did observe that girls were more likely to drink/eat 'tea and coffee' and 'butter' and less likely to eat 'meat, fish and eggs' for breakfast than boys, which concurs with results from other studies ${ }^{(10,24)}$.

Adolescents under the age of 15 years were more likely to be breakfast consumers and to consume more of the target food groups for breakfast than older adolescents. Aranceta et al. reported similar results in Spanish adolescents $^{(12)}$. Other authors have also shown that younger European adolescents consume breakfast more often than their older counterparts ${ }^{(1,10,13)}$. More autonomy and independence in regard to food choices among older adolescents could explain the age decline in breakfast consumption ${ }^{(25)}$. Younger adolescents were also more likely to drink 'other milk products' and less likely to consume 'tea and coffee', 'water' or 'added sugar' in comparison to older adolescents. This is in contrast to Raaijmakers et al. who showed that higher consumption from the liquid group was more common among older adolescents $^{(10)}$.

Adolescents from northern and central parts of Europe were more likely to score more highly on the breakfast quality index and were more likely to consume the target food groups than those from southern Europe. Risvas et al. suggested that Greek students have learnt to categorize food as 'healthier but less tasty' and 'appealing but unhealthy $^{(26)}$, while Finnish adolescents perceived e.g. healthy bread as pleasant and very acceptable ${ }^{(27)}$. These attitudinal differences to healthy food between countries might explain why we saw a higher score on the breakfast quality index among adolescents in the northern and central part of Europe.

We did not find significant differences in breakfast consumption $v$. skipping between the two regions in Europe. Vereecken et al. reported differences in the frequency of breakfast consumption among different countries in Europe; in the southern region daily breakfast consumption varied from 33\% (Greek girls) to $72 \%$ (Spanish boys), while in the northern/central region the daily breakfast consumption ranged from $42 \%$ (Hungarian girls) to $73 \%$ (Swedish boys) $^{(13)}$. Differences in breakfast consumption have also been reported between Greece and Finland ${ }^{(28)}$. It could be that many inter-country differences are even more pronounced than inter-regional differences, which may explain why we did not observe any differences in breakfast consumption between our two regions, but further studies are required to confirm this theory. 
Socio-economic status seems to be associated with dietary habits among adolescents but not to the same extent as in adults ${ }^{(29)}$. One reason for this could be that peers influence the adolescent's behaviour even more than does the family's socio-economic status. In our study, the only socio-economic variable that was associated with breakfast consumption was maternal education level; adolescents whose mothers had a high education level were more likely to be breakfast consumers compared with adolescents whose mothers had a low/medium education level. Similar results have been found in Norway ${ }^{(15)}$ and the USA ${ }^{(30,31)}$. Additionally, our study shows that adolescents whose mothers had a high education level were more likely to consume a breakfast of higher quality, to consume the target food groups and were less likely to consume 'soft drinks' for breakfast. Similar patterns have been shown in Norway ${ }^{(32)}$. Mothers have a strong influence on the dietary habits of their families and influence the family food environment, and mothers who are more educated may be more likely to consider health in their choice of foods ${ }^{(29,33)}$.

Adolescents reporting higher family affluence were more likely to consume a breakfast of higher quality, to consume the target food groups and to consume 'sweets', but less likely to consume 'other milk products', 'meat, fish and eggs' and 'vegetable fat' for breakfast in comparison to those reporting low family affluence. The HBSC Study showed an association between a high score on the FAS and breakfast consumption in five of the countries included in HELENA (Belgium, France, Germany, Hungary and Sweden) ${ }^{(13)}$. Higher fruit consumption among the more affluent adolescents has also been shown in the HBSC Study ${ }^{(34)}$. Less affluent families might have less money to spend on food ${ }^{(35)}$ and when cost is a barrier families might be less likely to consider health in their choice of food.

Finally, adolescents from a 'traditional' family structure were more likely to report a high-quality breakfast and were also more likely to eat 'sweets' for breakfast compared with those from single-parent/shared-care families. A similar association between breakfast consumption and this family structure were observed in the HBSC Study, among the countries included in HELENA, with the exception of Greece $^{(13)}$. Family cohesion has been found to be associated with breakfast consumption and decreased soda intake in a population of girls in the USA ${ }^{(30)}$. One possible explanation for the higher quality of breakfast in adolescents from traditional families could be that these adolescents could be more likely to comply with their parents' wishes regarding breakfast consumption and the family may be more likely to consume breakfast together.

\section{Strengths and limitations}

The strengths of our study include the large sample of adolescents. Our aim was to reach a sub-sample within each country that was representative of the whole population of adolescents in Europe regarding sex, age and socio-economic status. As it was compulsory in all countries for this age group to attend school and as we collected the data in large cities, we assert that the sample will be representative of large cities in Europe. The standardized and harmonized methodology is also a study strength. The computerized $24 \mathrm{~h}$ recall method provides both frequency and quality data about the breakfast and two non-consecutive $24 \mathrm{~h}$ recalls have shown to be a relatively reproducible estimate of the mean usual intake of a group ${ }^{(36)}$.

Some limitations of the current study include the fact that there is no accepted definition of breakfast in the literature; although the issue has been discussed at length $^{(11)}$, no consensus has been reached. For the purpose of our study we defined high and low breakfast quality based on a relatively simple index that considered both breakfast consumption and consumption of three desirable target food groups. The index does not allow for other food groups that may also be considered nutritious, nor can it take into account whether undesirable food groups were also consumed and in what quantities. The fact that the study population comes only from urban areas could mean that the sociodemographic distribution is not representative of the total population of the nine countries studied. The inability to compare the results between the different countries in Europe ${ }^{(17)}$ is also a limitation as heterogeneity in breakfast habits between European countries has been reported ${ }^{(13)}$. The $24 \mathrm{~h}$ recall was collected during school time on weekdays, so there are no Fridays and Saturdays in the dietary recall database; this could influence the results as differences in dietary intakes between weekdays and weekends have been reported ${ }^{(36)}$.

\section{Implications for further research}

The public health implications of poor breakfast consumption habits are considerable and our finding that only $4 \%$ of European adolescents consumed a high-quality breakfast highlights the need to promote breakfast, especially a high-quality breakfast, among adolescents. Particular attention should be paid to older adolescents, adolescents from the southern part of Europe and adolescents from families with low socio-economic status.

More specific information about the breakfast habits among European adolescents in larger country samples is needed to facilitate inter-country comparisons.

\section{Conclusions}

The majority of the adolescents consumed a 'low-quality' breakfast. Older adolescents, adolescents from the southern part of Europe (Greece, Italy and Spain) and adolescents from families with low socio-economic status were more likely to consume a 'low-quality' breakfast. 
The study highlights the need to promote 'high-quality' breakfast among adolescents, particularly to older adolescents, adolescents from southern Europe and adolescents from families with low socio-economic status.

\section{Acknowledgements}

The study took place with financial support from the European Community Sixth RTD Framework Programme (Contract FOOD-CT-2005-007034); the Swedish Council for Working Life and Social Research (FAS), the Swedish Heart-Lung Foundation (20090635); and the Spanish Ministry of Health: Maternal, Child Health and Development Network (number RG08/0072). The contents of this article reflect only the authors' views and the European Community is not liable for any use that may be made of the information contained therein. The writing group takes sole responsibility for the content of this article. None of the authors had a personal of financial conflict of interest. L.H., C.A.V., I.L. and J.R.R. wrote the manuscript and performed the statistical analysis; L.H., C.A.V., I.L., J.R.R., C.L.D., M.C.G., C.C.G., S.G.M., E.G., I.H., A.K., M.K., Y.M., D.M., E.P., K.W., T.D.V., L.A.M. and M.S. contributed to the interpretation and discussion of the results and critically revised the drafted manuscript. The authors thank all the adolescents who took part in the HELENA study.

\section{References}

1. Rampersaud GC, Pereira MA, Girard BL et al. (2005) Breakfast habits, nutritional status, body weight, and academic performance in children and adolescents. $J \mathrm{Am}$ Diet Assoc 105, 743-760.

2. Ruxton $\mathrm{CH} \&$ Kirk TR (1997) Breakfast: a review of associations with measures of dietary intake, physiology and biochemistry. Br J Nutr 78, 199-213.

3. Matthys C, De Henauw S, Bellemans M et al. (2007) Breakfast habits affect overall nutrient profiles in adolescents. Public Health Nutr 10, 413-421.

4. Sjöberg A, Hallberg L, Höglund D et al. (2003) Meal pattern, food choice, nutrient intake and lifestyle factors in The Goteborg Adolescence Study. Eur J Clin Nutr 57, $1569-1578$.

5. Hoyland A, Dye L \& Lawton CL (2009) A systematic review of the effect of breakfast on the cognitive performance of children and adolescents. Nutr Res Rev 22, 220-243.

6. Szajewska H \& Ruszczynski M (2010) Systematic review demonstrating that breakfast consumption influences body weight outcomes in children and adolescents in Europe. Crit Rev Food Sci Nutr 50, 113-119.

7. Dialektakou KD \& Vranas PB (2008) Breakfast skipping and body mass index among adolescents in Greece: whether an association exists depends on how breakfast skipping is defined. J Am Diet Assoc 108, 1517-1525.

8. O'Sullivan TA, Robinson M, Kendall GE et al. (2009) A good-quality breakfast is associated with better mental health in adolescence. Public Health Nutr 12, 249-258.

9. Wesnes KA, Pincock C, Richardson D et al. (2003) Breakfast reduces declines in attention and memory over the morning in schoolchildren. Appetite 41, 329-331.
10. Raaijmakers LG, Bessems KM, Kremers SP et al. (2010) Breakfast consumption among children and adolescents in the Netherlands. Eur J Public Health 20, 318-324.

11. Giovannini M, Verduci E, Scaglioni S et al. (2008) Breakfast: a good habit, not a repetitive custom. J Int Med Res 36, 613-624.

12. Aranceta J, Serra-Majem L, Ribas L et al. (2001) Breakfast consumption in Spanish children and young people. Public Health Nutr 4, 1439-1444.

13. Vereecken CA, Dupuy M, Rasmussen M et al. (2009) Breakfast consumption and its socio-demographic and lifestyle correlates in schoolchildren in 41 countries participating in the HBSC study. Int J Public Health 54, Suppl. 2, 180-190.

14. Croezen S, Visscher TL, Ter Bogt NC et al. (2009) Skipping breakfast, alcohol consumption and physical inactivity as risk factors for overweight and obesity in adolescents: results of the E-MOVO project. Eur J Clin Nutr 63, 405-412.

15. Lien L (2007) Is breakfast consumption related to mental distress and academic performance in adolescents? Public Health Nutr 10, 422-428.

16. Johansen A, Rasmussen S \& Madsen M (2006) Health behaviour among adolescents in Denmark: influence of school class and individual risk factors. Scand J Public Health 34, 32-40.

17. Moreno LA, De Henauw S, Gonzalez-Gross M et al. (2008) Design and implementation of the Healthy Lifestyle in Europe by Nutrition in Adolescence Cross-Sectional Study. Int J Obes (Lond) 32, Suppl. 5, S4-S11.

18. Beghin L, Castera M, Manios Y et al. (2008) Quality assurance of ethical issues and regulatory aspects relating to good clinical practices in the HELENA Cross-Sectional Study. Int J Obes (Lond) 32, Suppl. 5, S12-S18.

19. Iliescu C, Beghin L, Maes L et al. (2008) Socioeconomic questionnaire and clinical assessment in the HELENA Cross-Sectional Study: methodology. Int J Obes (Lond) 32, Suppl. 5, S19-S25.

20. Boyce W, Torsheim T, Currie C et al. (2006) The family affluence scale as a measure of national wealth: validation of an adolescent self-report measure. Soc Indic Res 78, 473-487.

21. Vereecken CA, Covents M, Sichert-Hellert W et al. (2008) Development and evaluation of a self-administered computerized 24-h dietary recall method for adolescents in Europe. Int J Obes (Lond) 32, Suppl. 5, S26-S34.

22. Vereecken CA, Covents M, Matthys C et al. (2005) Young adolescents' nutrition assessment on computer (YANA-C). Eur J Clin Nutr 59, 658-667.

23. Alexy U, Wicher M \& Kersting M (2010) Breakfast trends in children and adolescents: frequency and quality. Public Health Nutr 13, 1795-1802.

24. Von Post-Skagegard M, Samuelson G, Karlstrom B et al. (2002) Changes in food habits in healthy Swedish adolescents during the transition from adolescence to adulthood. Eur J Clin Nutr 56, 532-538.

25. Story M, Neumark-Sztainer D \& French S (2002) Individual and environmental influences on adolescent eating behaviors. J Am Diet Assoc 102, 3 Suppl., S40-S51.

26. Risvas G, Panagiotakos DB \& Zampelas A (2008) Factors affecting food choice in Greek primary-school students: ELPYDES study. Public Health Nutr 11, 639-646.

27. Pohjanheimo T, Luomala H \& Tahvonen R (2010) Finnish adolescents' attitudes towards wholegrain bread and healthiness. J Sci Food Agric 90, 1538-1544.

28. Veltsista A, Laitinen J, Sovio U et al. (2010) Relationship between eating behavior, breakfast consumption, and obesity among Finnish and Greek adolescents. J Nutr Educ Behav 42, 417-421.

29. Hanson MD \& Chen E (2007) Socioeconomic status and health behaviors in adolescence: a review of the literature. J Behav Med 30, 263-285. 
30. Franko DL, Thompson D, Bauserman R et al. (2008) What's love got to do with it? Family cohesion and healthy eating behaviors in adolescent girls. Int J Eat Disord 41, 360-367.

31. Timlin MT, Pereira MA, Story M et al. (2008) Breakfast eating and weight change in a 5 -year prospective analysis of adolescents: Project EAT (Eating Among Teens). Pediatrics 121, e638-e645.

32. Nilsen SM, Krokstad S, Holmen TL et al. (2010) Adolescents' health-related dietary patterns by parental socioeconomic position, the Nord-Trondelag Health Study (HUNT). Eur J Public Health 20, 299-305.
33. Pearson N, Biddle SJ \& Gorely T (2009) Family correlates of breakfast consumption among children and adolescents. A systematic review. Appetite 52, 1-7.

34. Vereecken CA, Inchley J, Subramanian SV et al. (2005) The relative influence of individual and contextual socio-economic status on consumption of fruit and soft drinks among adolescents in Europe. Eur J Public Health 15, 224-232.

35. Roos E, Prattala R, Lahelma E et al. (1996) Modern and healthy?: socioeconomic differences in the quality of diet. Eur J Clin Nutr 50, 753-760.

36. Gibson RS (2005) Principles of Nutritional Assessment, 2nd ed. New York: Oxford University Press. 